\title{
An enhanced hybrid Pareto metaheuritic algorithm-based multicast tree estimation for reliable multicast routing in VANETS
}

\author{
Sengathir Janakiraman \\ Department of Information Technology, CVR College of Engineering, Mangalpally, \\ Vastunagar, Rangareddy District Telangana, India-501510 \\ *Corresponding author, e-mail: j.sengathir@gmail.com
}

\begin{abstract}
Prompt and reliable data dissemination among the vehicular nodes of the network is indispensable as its mobility rate and limited coverage characteristics introduce the possibility of frequent topology changes. The effective and efficient sharing of critical information in the event of emergency necessitates either direct interaction or Road Side Units (RSUs)-based vehicular communication in the primitive place. Multicast routing is confirmed to be the significant scheme of data transfer since they establish reliable data dissemination between the source and destination vehicular nodes by estimating an optimal multicast tree. Moreover, QoS-constraint enforced meta-heuristic approaches are considered to be excellent for determining optimal multicast tree under multicasting. An Enhanced Hybrid Pareto Metaheuritic Algorithm-based Multicast Tree Estimation Scheme (EHPMA-MTES) is contributed for reliable multicast routing. The proposed EHPMA-MTES is confirmed to reduce the cost of transmission by $28 \%$ through the minimization of the multicast tree count formed during the process of multicast routing.
\end{abstract}

Keywords: emergency information, multicast routing, optimal multicast tree, Pareto metaheuritic algorithm, road side units

Copyright $@ 2018$ APTIKOM - All rights reserved.

\section{Introduction}

The Intelligent Transportation Systems (ITSs) are considered as the greatest boon to the vehicular network domain since they focus and utilize on the mobility features of vehicular nodes for facilitating predominant safety on the roads [1]. The ITSs are responsible for ensuring ambience to the vehicular network user from sending and receiving sensitive messages that prevents them from collision avoidance and accidents [2]. The multicasting process of sensitive information exchange is identified to be the excellent method of transmitting data to multiple number of vehicles in the network [3]. But, these exchanges of sensitive information from the source to the destination nodes are established based on the estimation of multiple multicast trees. However, one of the optimal multicast tree must be determined for effective data dissemination by reducing the QoS factors of jitter, delay and cost with enhanced bandwidth utilization rate [4]. Further, the measure of optimality can be potentially determined in the process of multicast routing by devising multi-objective function that estimates the impact of QoS factors that are influential in the act of multicasting [5]. A number of meta-heuristic mechanisms based on traditional Ant Colony Optimization (ACO), Particle Swarm optimization (PSO) and Artificial Bee Colony Optimization (ABC) approaches are contributed for optimal selection of multicast tree that in turn enhances the rate of effective data dissemination [6]. But, most of these meta-heuristic approaches fail to estimate solutions without early convergence and superior spread in the process of identifying the optimal multicast tree under the event of multicasting.

The proposed EHPMA-MTES approach is proposed as an effective optimal multicast tree estimation approach for eliminating the issue of the delayed convergence and inferior spread that are common in vehicular network during the process of multicasting even when the influential QoS factor are imposed. This proposed EHPMA-MTES scheme uses an additional step for determining the Pareto optimal front factor that aids in rapid and precise multicasting process with the help of an optimal multicast tree. The experimental evaluation of the proposed EHPMA-MTES scheme was also facilitated based on the computation of fitness value derived using jitter, delay, cost and utilized bandwidth under a different number of monotonically increasing generations. 


\section{Related Work}

In this section, some of the most recent optimal multicast tree estimation approaches that facilitate better data dissemination in vehicular networks are discussed with the merits and limitations. The first among the recent optimal multicast tree estimation scheme is the Ant Colony Optimization-based Multicast Routing Technique (ACOMRT) that utilizes dynamic exploration and exploitation during the process of determining optimal multicast tree [7]. This ACOMRT was determined to enable better spread with reduced number of generations. The packet loss, jitter and delay of this ACOMRT was determined to be highly minimized based on the process of potentially preventing the limitations in the stagnation process. Then, Tabu Search-based optimal multicast tree estimation mechanism was propounded for dynamically increasing or decreasing the rate of exploration capability [8]. The number of multicast groups explored for optimal selection was also proved to relatively low compared to the ACO and PSO schemes of the literature. The packet delivery rate and throughput of this tabu search algorithm was determined to be excellent under different thresholds used for enforcing QoS factors during implementation. Further, a Harmony Searchbased optimal multicast tree estimation technique was proposed for preventing the drawbacks of the previous ACO and PSO schemes [9]. This Harmony Search-based approaches were detected to be superior in exploring the search domain in order to restrict the earlier convergence to a non-optimal point of optimization. This Harmony Serach process is also confirmed to reduce energy consumptions, packet loss and delay in the superior multicast tree estimation process. Further, a Bee life-based optimal multicast tree estimation technique was proposed for reducing the rate of normalized load and packet latency under multicasting. This Bee life-based multicasting approach utilized the merits of partial zone in order to minimize the hurdles that are more possible under multicasting process [10]. Another improved Bee Lifebased optimal multicast tree estimation technique was proposed for reducing the degree of packet latency and energy consumptions by eliminating the problem of QoS condition imposition through the incorporation of appropriate tradeoff between the extent of intensification and diversification [11]. In addition, an Artificial Bee Colony (ABC)-based optimization scheme was proposed for eliminating the limitations of ACO and Tabu-based multicast tree optimization mechanisms [12]. The main drawback identified with this $\mathrm{ABC}$ mechanism is the slow rate of convergence and raid falling of the solution into a non-optimal point that could not yield optimal results. The packet delivery rate, throughput and number of multicast tree optimized are determined to be excellent in this ABC-based multicast tree optimization mechanism since they are capable in precise conclusion of optimal tree that reduces the rate of packet loss in the network.

\section{Proposed Enhanced Hybrid Pareto Metaheuritic Algorithm-based Multicast Tree Estimation Scheme (EHPMA-MTES)}

In this proposed EHPMA-MTES approach, the vehicular network on which the effective multicasting process needs to be facilitated is modeled as a weighted directional graph in which each vehicle of the network and connectivity between vehicles is considered as vertices and edges. The connectivity established between the vehicular nodes during data dissemination is estimated based on utilizing bandwidth, cost, jitter and delay such that the optimal multicast path from the network can be determined more effectively. Thus, this proposed EHPMA-MTES scheme represents a minimizing objective optimization issue that focuses on potential estimation of multicast tree that reduces the utilizing bandwidth, cost, jitter and delay when the path of the multicast tree is followed during the process of group communication in vehicular networks. The thresholds of jitter $\left(\operatorname{Jitter}_{T H}\right)$, delay $\left(\right.$ delay $\left._{T H}\right)$ and utilizing bandwidth $\left(B W_{T H}\right)$ is also assigned for understanding its impact during the process of estimating optimal multicast tree through the paths specified through $(p(s, t))$. Further, priorities $p_{1}, p_{2}, p_{3}$ and $p_{4}$ are used for projecting the influence of each factor ovr the estimation of optimal multicast tree. Thus, this optimization that concentrates on the reduction of the utilizing bandwidth, cost, jitter and delay on the multicast tree estimated under efficient routing process is expressed in (1) with conditions portrayed in (2)(5) respectively.

$$
\text { Minimize } f\left(O_{M T}(s, d)\right)=p_{1} * f_{\text {jitter }}+p_{2} * f_{\text {cos } t}+p_{3} * f_{\text {delay }}+p_{4} * f_{B W}
$$

Subject to the multiple constraints 


$$
\begin{aligned}
& f_{\text {jitter }}=\operatorname{Maximum}\left(\sum_{t-1}^{M(t)} \operatorname{Jitter}\left(p(s, t) \leq \operatorname{Jitter}_{T H}\right)\right. \\
& f_{\cos t}=\cos t\left(O_{M T}(s, d)\right) \\
& f_{\text {delay }}=\operatorname{Maximum}\left(\sum_{t-1}^{M(t)} \operatorname{delay}\left(p(s, t) \leq \operatorname{delay}_{T H}\right)\right. \\
& f_{B W}=\operatorname{Minimum}\left(\sum_{t-1}^{M(t)} B W\left(p(s, t) \leq B W_{T H}\right)\right.
\end{aligned}
$$

This proposed EHPMA-MTES approach is an improved version of Strength Pareto Optimization Scheme that focuses on superior spread and convergence under least number of generations during the process of determining the optimal path when the multiple constraints of QoS are imposed during the routing activity. This utilized enhanced version of the Strength Pareto Optimization Scheme comprised of seven steps that includes initialization, fitness estimation, environment factor selection, termination, selection of a mate, variation estimation and Pareto optimal front determination. This multi-objective optimization problem of multicast tree determination is considered to possess a number of multicast trees $\left(V_{1}, V_{2}, V_{3}, \ldots, V_{(m)}\right)$ from which optimal multicast tree needs to be estimated for cost effective data dissemination with reduced jitter, delay, and maximized utilized bandwidth. Then, the Pareto Front point is determined based on interpolation variable with a predefined number of generations. The number of generations is fixed prior to the process of determining an optimal multicast tree since the proposed EHPMA-MTES approach focuses on reducing the number of generations predominant to the exiting Bee life and $\mathrm{ABC}$ approaches.

$$
\begin{aligned}
& \lambda_{\text {Step }(j)}=\frac{\left|V_{(j)}-V_{(j)}^{U L}\right|}{D_{N}} \\
& V_{L}=V_{(j)}+\lambda_{S t e p(j)} \\
& V_{U}=V_{(j)}-\lambda_{\text {Step }(j)}
\end{aligned}
$$

Where ' $D_{N}$ ' represents the divisions count and $\lambda_{\text {Step }(j)}$ called as the variable of interpolation.

In this proposed EHPMA-MTES approach, the degree of diversification depends on the number of divisions count with reduced convergence rate. Thus, the deviation between the degree of exploration and exploitation is maintained by assigning the number of divisions to 2 to 20 . In the application of proposed EHPMA-MTES approach in the multicasting process, the method of string encoding is used for representing the distinct paths that exist between the source and destination with the aid of intermediate nodes. Thus, the optimal multicast tree is determined after the predefined number of generations that are really potential. In the next section, the steps involved in the implementation of the proposed EHPMAMTES Scheme is discussed.

\footnotetext{
Algorithm: Proposed EHPMA-MTES Scheme

Input: Set of multicast tree feasible to be constructed from the network for establishing reliable multicasting between the source and destinations.

Output: A distinct optimal multicast tree for multicasting that satisfies the QoS constraints of reduced jitter, delay and cost with reduced bandwidth utilization.

Step 1: Estimate the step of interpolation depending on the number of multicast trees that need to be considered for optimization.
}

APTIKOM J. CSIT Vol. 3, No. 3, 2018: 100-105 
Step 2: The decision variable that estimates a multicast tree as the optimal multicast tree is dynamically investigated based on (7) and (8)

Step 3: Apply the strategy of acceptance for selecting a optimal multicast using the computed Pareto Front point derived based on (1).

Step 4: Compute the correlation degree between the previous fitness value of a multicast tree with its current fitness value.

Step 5: If the previous fitness of the solution is lesser than current fitness value, then replace the previous fitness value is replaced with the lower limit of fitness forecasted value determined using the Grey theory. Step 6: Else, replace the previous fitness value is replaced by the upper limit of fitness forecasted value.

In the forthcoming section, the predominant performance of the proposed EHPMA-MTES approach is investigated based on the fitness value computed for jitter, delay, cost and utilized bandwidth under a varying number of generations.

\section{Simulation Results and Analysis}

The simulation experiments of the proposed EHPMA-MTES approach are conducted for investigating its predominance using ns-2.32 that runs on the Linux Ubunty 10.04 operating system. The implementation code of the proposed EHPMA-MTES are written in $\mathrm{C}++$ since the possibility of combining the implemented code with the routing protocol is optimal. In the utilized mobility model used for implementing the proposed EHPMA-MTES approach, the vehicles are considered to move with the velocity ranging from $5 \mathrm{~m} / \mathrm{sec}$ and $25 \mathrm{~m} / \mathrm{sec}$ respectively. The number of vehicular nodes in the topology considered for the implementation of the proposed EHPMA-MTES approach is 200 with nodes 5,15,25,45 and 65 considered as the multicast leader node. The terrain area, simulation time and the base protocol of the proposed EHPMA-MTES approach is 1500x1500 square meters, 200 seconds and DSR protocol. The priorities and threshold limits of delay, jitter and utilized bandwidth are assigned to 1,10,1 and, $1200 \mathrm{~m} / \mathrm{sec}, 15 \mathrm{~m} / \mathrm{sec}$ and 600 kilobits per seconds respectively.

In this experimental investigation, the significance of the proposed EHPMA-MTES approach is evaluated based on the fitness value of jitter, delay, cost and multicast groups. Figure 1 presents the predominance of the proposed EHPMA-MTES scheme evaluated based on jitter-based fitness value. The fitness value of the proposed EHPMA-MTES technique computed based on jitter is comparatively low compared to the BLMRT, ABCMRT and ACOMRT considered for investigation since it facilitates better spread and intensification during the process of estimating the optimal multicast during the process of multicasting. The fitness value determined based on jitter for the proposed EHPMA-MTES scheme is only 7.63, while it is 9.34, 9.58 and 9.83 for the comparable BLMRT, ABCMRT and ACOMRT approaches. Similarly, Figure 2 portrays the excellence of the proposed EHPMA-MTES scheme evaluated based on delay. The fitness value of the proposed EHPMA-MTES technique computed based on delay is also less than the comparable BLMRT, ABCMRT and ACOMRT schemes due the flexibility factor that determines the rate of adjustment that are essential in the process of estimating optimal multicast tree for data transmission. The delay-based fitness value for the proposed EHPMA-MTES scheme is only 510.24 when it is $810.23,870,45$ and 890.56 for the comparable BLMRT, ABCMRT and ACOMRT approaches.

Figure 3 unveils the potential of the proposed EHPMA-MTES scheme evaluated based on transmission cost based fitness value. The fitness value of the proposed EHPMA-MTES technique computed based on sot of transmission is reduced since it incorporates a phenomenal mating step for eliminating the overheads that are possible under the multicasting routing activity. The fitness value determined based on transmission cost for the proposed EHPMA-MTES scheme is only 1598.245, even when it is 2100.23,2222.34 and 2235.435 for the comparable BLMRT, ABCMRT and ACOMRT approaches. In addition, Figure 4 exemplars the potential of the proposed EHPMA-MTES scheme evaluated based on multicast group fitness value. The fitness value of the proposed EHPMA-MTES technique computed based on multicast groups is the least compared to the BLMRT, ABCMRT and ACOMRT considered for investigation since it used an additional step for improving the accuracy in estimating the optimal multicast during the event of multicasting. The fitness value determined based on multicast group for the proposed EHPMA-MTES scheme is only 15567.23, while it is 20156.702, 21233.713 and 22234.56 for the comparable BLMRT, ABCMRT and ACOMRT approaches. 


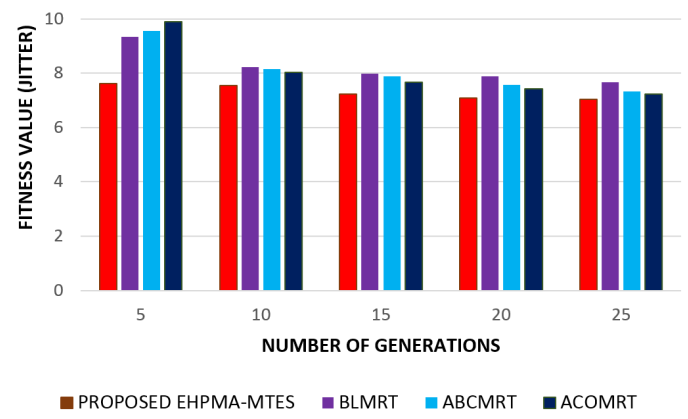

Figure 1. Investigation of the proposed EHPMA-MTES-Fitness Value (Jitter)

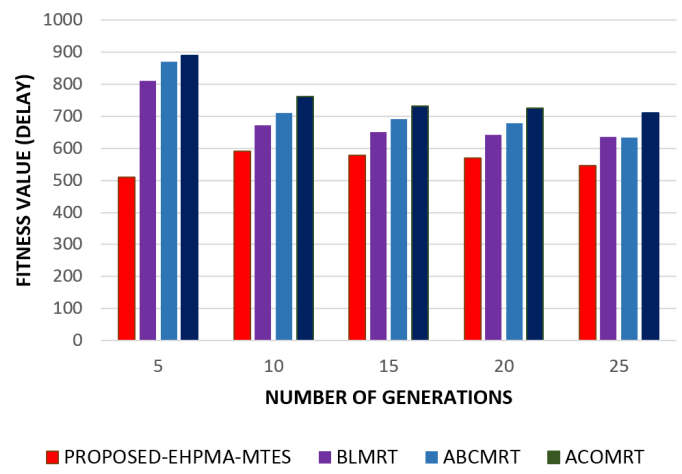

Figure 2. Investigation of the proposed EHPMA-MTES-Fitness Value (Delay)

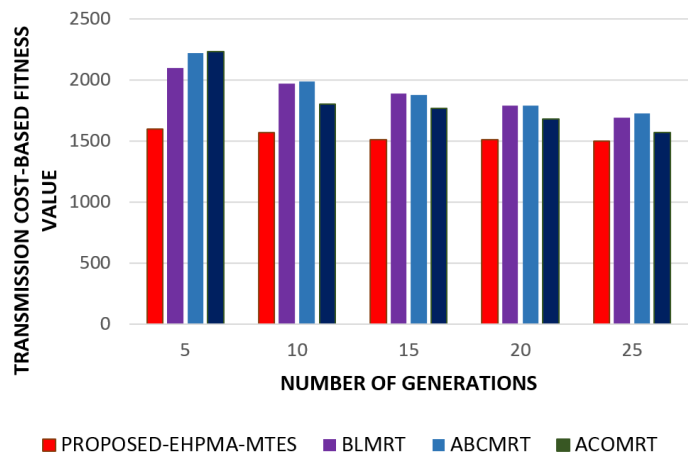

Figure 3. Investigation of the proposed EHPMA-MTES-Fitness (Cost)

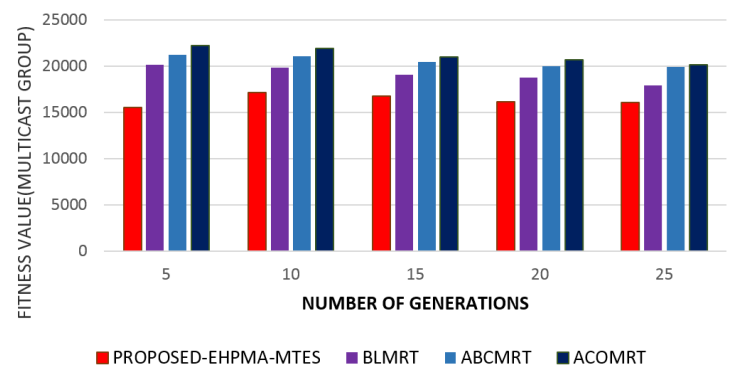

Figure 4. Proposed EHPMA-MTES-Fitness Value (Multicast Group) 


\section{Conclusion}

The proposed EHPMA-MTES was presented as the reliable meta-heuristic approach-based multicast routing scheme that derives the merits of Pareto optimization process for ensuring reliable data dissemination. This proposed EHPMA-MTES was also contributed to reduce the cost of transmission by minimizing the latency and jitter with enhanced bandwidth utilization during the process of multicasting. The simulation experiments of the proposed EHPMA-MTES scheme facilitated with a fitness value manipulated using multicast tree, jitter and latency was confirmed to be significant on par with the existing MQBV-MTES, BLMC-MTES and HSA-MTES approaches. The fitness value of the transmission cost enabled by the proposed EHPMA-MTES approach was confirmed to be remarkable with outstanding value of 1689.455 which is superior to the fitness value of $1768.342,1723.564$ and 1892.546 in transmission cost ensured by the benchmarked MQBV-MTES, BLMC-MTES and HSA-MTES approaches. The fitness value determined based on multicast group for the proposed EHPMA-MTES scheme is approximately $21 \%, 16 \%$ and $12 \%$ superior to the comparable BLMRT, ABCMRT and ACOMRT approaches used for investigation. As the part of future research, it is planned to devise a ACO-ABC algorithm -based multicast tree estimation process in order to understand the significance behind the process of integrating the exploration ability of ants and exploitation potential of artificial bees.

\section{References}

[1] Wang, H, Xu, H, Yi, S, Shi, Z. A tree-growth based ant colony algorithm for QoS multicast routing problem. Expert Systems with Applications. 2011; 38(9): 11787-11795.

[2] Tseng, S, Lin, C, Huang, Y. Ant colony-based algorithm for constructing broadcasting tree with degree and delay constraints. Expert Systems with Applications. 2008; 35(3): 1473-1481.

[3] Bitam, S, Mellouk, A. Routing for Vehicular Ad Hoc Networks. Bio-Inspired Routing Protocols for Vehicular Ad Hoc Networks. 2014; 2(1): 29-50.

[4] Armaghan, M, Haghighat, AT, Armaghan, M. QoS multicast routing algorithms based on Tabu Search with Elite candidate list. 2009 International Conference on Application of Information and Communication Technologies, 2009; 2(1): 77-86.

[5] Sun, J, Fang, W, Wu, X, Xie, Z, Xu, W. QoS multicast routing using a quantum-behaved particle swarm optimization algorithm. Engineering Applications of Artificial Intelligence. 2011; 24(1): 123-131.

[6] Yen, Y, Chao, H, Chang, R, Vasilakos, A. Flooding-limited and multi-constrained QoS multicast routing based on the genetic algorithm for MANETs. Mathematical and Computer Modelling. 2011; 53(11-12): 2238-2250.

[7] Haghighat, A, Faez, K, Dehghan, M, Mowlaei, A, Ghahremani, Y. GA-based heuristic algorithms for bandwidthdelay-constrained least-cost multicast routing. Computer Communications. 2004; 27(1): 111-127.

[8] Ghaboosi, N, Haghighat, AT. Tabu search based algorithms for bandwidth-delay-constrained least-cost multicast routing. Telecommunication Systems. 2007; 34(3-4): 147-166.

[9] Forsati, R, Haghighat, A, Mahdavi, M. Harmony search based algorithms for bandwidth-delay-constrained leastcost multicast routing. Computer Communications. 2008; 31(10): 2505-2519.

[10] Bitam, S, Mellouk, A. Bee life-based multi constraints multicast routing optimization for vehicular ad hoc networks. Journal of Network and Computer Applications. 2013; 36(3): 981-991.

[11] Bitam, S, Mellouk, A, Fowler, S. MQBV: multicast quality of service swarm bee routing for vehicular ad hoc networks. Wireless Communications and Mobile Computing. 2013; 15(9): 1391-1404.

[12] Zhang, X, Zhang, X, Gu, C. A micro-artificial bee colony based multicast routing in vehicular ad hoc networks. Ad Hoc Networks. 2017; 58: 213-221. 